\title{
Interação entre Terapeutas Comportamentais e Clientes Diagnosticados com Transtorno Obsessivo-Compulsivo
}

\author{
Denis Roberto Zamignani ${ }^{1}$ \\ Maria Amalia Pie Abib Andery \\ Pontifícia Universidade Católica de São Paulo
}

\begin{abstract}
RESUMO - A presente pesquisa teve como objetivo caracterizar a atuação verbal de dois terapeutas comportamentais no atendimento de clientes com o diagnóstico de transtorno obsessivo-compulsivo (TOC). Foram gravadas e transcritas de quatro a cinco sessões de atendimento com cada terapeuta atendendo um cliente. As verbalizações foram classificadas segundo a presença ou ausência de verbalizações de queixa, em categorias de descrição, explicação, aconselhamento, feedback, inferência, perguntas e outras verbalizações. A análise dos dados indicou que o terapeuta A "conseqüenciava" diferencialmente determinadas classes de verbalizações do cliente, teve percentual elevado de verbalizações de aprovação e teve uma ocorrência pequena de verbalizações de aconselhamento. Já o terapeuta B apresentou predominantemente verbalizações de aconselhamento e explicação e sua intervenção teve como foco principal a queixa do cliente. Ambos os terapeutas tenderam a dar explicações com ênfase em relações resposta-conseqüência, o que sugere coerência com os pressupostos da análise do comportamento.
\end{abstract}

Palavras-chave: terapia comportamental; transtorno obsessivo-compulsivo; relação terapêutica.

\section{Interaction between Behavior Therapists and their Clients Diagnosed with Obsessive-Compulsive Disorder}

\begin{abstract}
This study aimed at characterizing the work of two behavior analysts in a therapeutic setting, with clients with a diagnosis of obsessive-compulsive disorder (OCD). Four to five therapeutic sessions of each pair (therapist - client) were recorded. The therapist and clients' verbalizations were classified in relation to their relation to the client's complaints, and were categorized as: questions, descriptions, explanations, advices, feedback, inferences or other verbalization. Results indicated differences between the two therapists: the verbalization of therapists A suggested that he applied differential reinforcement to certain classes of client's verbalizations, with a small occurrence of counseling, while the verbalization of therapist B was classified as giving advice and explaining. For both therapists, the majority of their explanations about the clients' behavior were classified as emphasizing the response-consequence relationship, whichy be suggestive of coherence with the behavior analytical approach.
\end{abstract}

Key words: behavioral therapy; obsessive-compulsive disorder; therapeutic relationship.

Os modelos vigentes de nomenclatura psiquiátrica (DSM, 1995; OMS, 1993) estabelecem critérios diagnósticos para os chamados transtornos psiquiátricos, que são definidos pelo conjunto de (topografias de) comportamentos considerados típicos para determinados quadros clínicos. A avaliação do comportamento tendo em vista exclusivamente critérios topográficos, no entanto, é incompatível - ou, no mínimo, insuficiente - quando o profissional visa uma análise das contingências responsáveis pela instalação e manutenção do problema apresentado pelo cliente, como é o caso do analista do comportamento.

Em muitos trabalhos de autores de abordagem comportamental ou cognitivo-comportamental (Freeston \& Ladouceur, 2003; Salkovskis \& Kirk, 1997), o comportamento obsessivo-compulsivo é interpretado basicamente como um comportamento de esquiva. O procedimento de exposição com prevenção de respostas, que consiste no enfrentamento sistemático e gradual dos estímulos chamados

1 Endereço: Laboratório de Psicologia Experimental, Rua João Ramalho, 301, Perdizes, São Paulo, SP, Brasil 05008-001.

E-mail: dzamig@terra.com.br ansiogênicos (Riggs \& Foa, 1999), seria então recomendado como a principal estratégia de intervenção. Assim, autores como Salkovskis e Kirk (1997) e Freeston e Ladouceur (2003) propõem como critério para a escolha das técnicas de intervenção o próprio diagnóstico psiquiátrico e utilizam basicamente procedimentos padronizados.

$\mathrm{Na}$ literatura sobre o transtorno obsessivo-compulsivo (TOC), em especial nos manuais de atendimento a transtornos psiquiátricos, mesmo aqueles voltados para as chamadas terapias comportamentais (Barlow, 1999; Caballo, 2003), há uma excessiva ênfase em técnicas e procedimentos, a despeito de uma análise das contingências envolvidas no caso individual. Embora o uso de técnicas seja de fato parte da prática dos terapeutas comportamentais que se intitulam analistas do comportamento, sua aplicação de forma padronizada e universal quando este se defronta com certos padrões topográficos de comportamento, em detrimento de uma análise individual, é questionada por alguns autores (Banaco, 1999; Banaco \& Zamignani, 2004; Meyer, 1995; Vermes \& Zamignani, 2002).

Devido a questões deste tipo envolvidas na sua prática, é possível que o terapeuta comportamental encontre dificuldade para desenvolver o seu trabalho de forma coerente com os 
seus pressupostos teóricos, especialmente ao atender clientes com este tipo de queixa. A utilização de critérios topográficos para a identificação do problema, bem como a utilização de técnicas padronizadas para seu tratamento não são recursos consistentes com uma prática que enfatiza o caráter idiossincrático do responder de cada cliente e, conseqüentemente, da análise de contingências a ser desenvolvida.

Enquanto críticos da aplicação generalizada de técnicas e procedimentos padronizados, é importante que, em contrapartida, os terapeutas analistas do comportamento tenham à sua disposição um conjunto de estratégias de intervenção que viabilizem o atendimento a esse tipo de população, já que existe uma demanda para tal atendimento. Essas estratégias, entretanto, devem responder à exigência de uma análise individual, consistente com os pressupostos da análise do comportamento.

Meyer (1995), baseada no trabalho de Hayes (1991), listou alguns requisitos (níveis de análise) que deveriam conduzir a análise do trabalho do terapeuta para considerá-lo compatível com a abordagem comportamental. No nível tecnológico, seria avaliado se o conjunto de técnicas e procedimentos empregados pelo terapeuta é derivado de estudos realizados por pesquisadores da análise do comportamento. No nível metodológico, avaliar-se ia se a análise funcional ${ }^{2}$ é aspecto fundamental do trabalho terapêutico. No nível conceitual, Meyer (1995) afirma que seria necessária a avaliação do conhecimento dos princípios básicos do comportamento por parte do terapeuta, assim como a relação desses princípios com as práticas (técnicas e procedimentos) empregadas pelo terapeuta. O nível filosófico conduziria o terapeuta a avaliar se ele assume os pressupostos filosóficos do behaviorismo radical.

A questão levantada no presente trabalho, portanto, foi se os problemas apontados na classificação diagnóstica e na literatura clínica de casos de Transtorno ObsessivoCompulsivo poderiam levar a uma inconsistência na prática do terapeuta com relação aos pressupostos teóricos por ele adotados, quando do atendimento a essa população. Em vista disso, teve-se como objetivo a caracterização da prática verbal (vocal) de dois terapeutas autodenominados analistas do comportamento no atendimento de dois clientes com o diagnóstico de transtorno obsessivo-compulsivo. Por meio desta caracterização, pretendeu-se identificar elementos da prática destes terapeutas no atendimento de clientes com este diagnóstico que fossem consistentes (ou não) com os pressupostos da análise do comportamento.

Vale ressaltar que no trabalho original, cada um dos dois terapeutas foi estudado atendendo um cliente que apresentava diagnóstico de TOC e outro com outro tipo de queixa, de forma que cada terapeuta pudesse ser comparado com ele mesmo nas diferentes condições. Entretanto, por uma questão de espaço, o presente artigo omite os dados relativos aos clientes sem diagnóstico de TOC.

2 No presente artigo, será dada preferência ao uso do termo Análise de Contingências, em substituição ao termo Análise Funcional, pois considera-se que o termo Análise Funcional é corretamente aplicado apenas em situações que envolvem a descrição de relações funcionais experimentalmente demonstradas, sendo inadequado seu uso para a descrição de inferências clínicas. O termo análise funcional será utilizado com relação a descrição de relações entre eventos na clínica apenas em citações de autores que utilizaram originalmente esse termo.

\section{Método}

\section{Participantes}

Foram participantes deste trabalho (a) dois terapeutas autodenominados analistas do comportamento, cada um deles atendendo um cliente diagnosticado por um médico psiquiatra como portador de TOC. A formação dos terapeutas foi realizada em universidades de diferentes estados da União. $\mathrm{O}$ cliente atendido pelo terapeuta A estava há mais de um ano em atendimento, enquanto o cliente do terapeuta B estava nas sessões iniciais. Clientes e terapeutas foram informados sobre o trabalho e deram seus consentimentos por escrito e o projeto foi aprovado pelo comitê de ética da PUC-SP.

\section{Material e Setting}

Foram utilizados um gravador de áudio para gravação das sessões e um toca-fitas para a transcrição dos dados da sessão. Para a transcrição e análise dos dados foi utilizado um computador, equipado com o software Etnograph $5.0^{3}$, desenvolvido especificamente para a análise qualitativa de documentos escritos, que permite a inserção e edição de texto, a criação e definição de categorias e sua inserção no texto; permite também a inserção de comentários e algumas análises estatísticas básicas, além da emissão de relatórios de análises de dados em versão impressa ou de arquivo de texto.

As sessões do terapeuta A foram gravadas na casa do cliente ou em outros ambientes variados (rua, carro, lojas, bares etc.) devido às atividades terapêuticas serem propostas nesses ambientes. Já as sessões do terapeuta B foram gravadas nas salas de atendimento da clínica-escola da PUC-SP, cedida pela Universidade para a realização da pesquisa.

\section{Procedimentos}

a) Coleta de dados: foram gravadas cinco ou mais sessões de cada díade. Das sessões do terapeuta A, foram selecionadas para análise, e transcritas, quatro sessões consecutivas. Já com o terapeuta B foram gravadas todas as sessões, desde a primeira entrevista e foram selecionadas para análise, e transcritas, cinco sessões. As sessões do terapeuta B ocorreram em intervalo de uma semana, e as do terapeuta A ocorriam três vezes por semana.

b) Preparação do material para análise: todas as transcrições foram revistas pelo pesquisador, omitindo-se as informações que identificavam os participantes e identificando-se verbalizações nas quais o terapeuta era o falante e verbalizações nas quais o cliente era o falante. Considerou-se uma verbalização tudo o que um participante dizia entre as falas do outro participante, independentemente do número de períodos gramaticais.

c) Preparação para categorização: para a categorização das verbalizações foram criadas categorias mutuamente exclusivas de registro do comportamento vocal, sendo 12 (doze) para o terapeuta e 14 para o cliente, conforme consta no quadro $1^{4}$. Algumas destas categorias especi-

3 Informações sobre o software no site http:/qualisresearch.com/

4 Definições mais completas e exemplos de verbalizações categorizadas podem ser localizados na dissertação do primeiro autor. 
Quadro 1. Lista de categorias de registro das verbalizações de terapeuta (T) e cliente (C)

\begin{tabular}{|c|c|c|c|}
\hline $\begin{array}{c}\text { Quem } \\
\text { verbaliza }\end{array}$ & Classe de Verbalização & Tema & Sigla \\
\hline $\mathrm{T}$ & \multirow{2}{*}{ Descreve } & \multirow{2}{*}{ eventos relacionados à queixa } & TDESQUE \\
\hline $\mathrm{C}$ & & & CDESQUE \\
\hline $\mathrm{T}$ & \multirow{2}{*}{ Descreve } & \multirow{2}{*}{ eventos diversos } & TDESDIV \\
\hline $\mathrm{C}$ & & & CDESDIV \\
\hline $\mathrm{T}$ & \multirow{2}{*}{ Descreve } & \multirow{2}{*}{ relações explicativas ou causais } & TDESEXP \\
\hline $\mathrm{C}$ & & & CDESEXP \\
\hline $\mathrm{C}$ & Descreve & melhora relativa à queixa & CDESMLHORA \\
\hline $\mathrm{T}$ & \multirow{2}{*}{ - Pergunta } & \multirow{2}{*}{ eventos relacionados à queixa } & TPERQUE \\
\hline $\mathrm{C}$ & & & CPERQUE \\
\hline $\mathrm{T}$ & \multirow{2}{*}{ - Pergunta } & \multirow{2}{*}{ eventos diversos } & TPERDIV \\
\hline $\mathrm{C}$ & & & CPERDIV \\
\hline $\mathrm{C}$ & Pergunta & pedindo conselhos para o terapeuta & CPERCONS \\
\hline $\mathrm{T}$ & Aconselha cliente ou sugere atividades & & TACO \\
\hline $\mathrm{T}$ & \multirow{2}{*}{ - Aprova - expressa julgamento favorável ou concordância } & & TFBAPR \\
\hline $\mathrm{C}$ & & & CFBAPR \\
\hline $\mathrm{T}$ & \multirow{2}{*}{ - Reprova - expressa julgamento não-favorável ou discordância } & & TFBREP \\
\hline $\mathrm{C}$ & & & CFBREP \\
\hline $\mathrm{C}$ & Expressa dúvida quanto a análise ou sugestão do terapeuta & & CFBDUV \\
\hline $\mathrm{T}$ & \multirow{2}{*}{ - Verbalizações Mínimas do tipo "hm hm”, “sei...” } & & TFBVM \\
\hline $\mathrm{C}$ & & & CFBVM \\
\hline $\mathrm{T}$ & Infere - supõe a ocorrência de eventos até então não relatados/observados & & TINF \\
\hline $\mathrm{T}$ & \multirow{2}{*}{ - Outras verbalizações } & & TOUT \\
\hline $\mathrm{C}$ & & & COUT \\
\hline $\mathrm{T}$ & \multirow{2}{*}{ Verbalizações com Registro Insuficiente } & & TREGINS \\
\hline $\mathrm{C}$ & & & CREGINS \\
\hline $\mathrm{C}$ & Engaja-se em comportamento obsessivo-compulsivo & & CENGTOC \\
\hline
\end{tabular}

ficavam se as verbalizações eram relacionadas à queixa ou a eventos não diretamente relacionados à queixa do cliente - chamados de "eventos diversos". Como o padrão de respostas de cada cliente era bastante diferente, foram considerados "queixa" todas as referências aos eventos relacionados ao padrão obsessivo-compulsivo que cada cliente apresentava, além de outros padrões de comportamento que o cliente houvesse descrito como alvo da terapia.

d) Categorização das sessões: as verbalização do terapeuta e do cliente foram classificadas e, quando necessário, subdivididas em segmentos. Considerava-se um segmento um trecho de verbalização classificável conforme uma determinada categoria. Um segmento terminava quando se iniciava uma nova categoria do mesmo falante na mesma verbalização, ou quando o próximo falante iniciava sua fala. Cada segmento identificado foi classificado, assim, exclusivamente em uma determinada categoria.

e) Teste de concordância entre observadores da categorização: foi feito um teste de concordância entre observadores da seleção dos segmentos, bem como de sua categorização propriamente dita, utilizando-se um avaliador independente, que foi treinado pelo pesquisador para este fim. Este observador categorizou uma única sessão do terapeuta $\mathrm{A}$, escolhida ao acaso. Para o cálculo do índice de acordo foi utilizada a seguinte fórmula:

$$
\mathrm{F}=\frac{\left(\mathrm{N}^{\mathrm{o}} \text { de linhas concordantes } \mathrm{x} 100\right)}{\mathrm{N}^{\mathrm{o}} \text { total de linhas da sessão }}
$$

As categorias cujo índice de concordância foi menor que $80 \%$ foram então redefinidas e o treino dos avaliadores e teste de concordância entre observadores refeitos. As porcentagens de acordos finalmente obtidas foram entre $82,3 \%$ e $92,1 \%$ para todas as categorias.

f) Análises realizadas por meio do software Etnograph: após a categorização de todas as verbalizações das sessões, foram registrados o número de linhas ocupadas e o número total de segmentos categorizados para cada um dos participantes, por sessão. O número de linhas ocupadas por verbalizações do terapeuta e do cliente foi escolhido como a unidade principal de quantificação das verbalizações ocorridas na sessão, por ser considerado mais representativo da ocorrência do comportamento verbal dos participantes ao longo do tempo. Um registro das ocorrências e percentual de verbalizações do terapeuta que se seguiram a verbalizações "Cliente Descreve Queixa" (CDESQUE) e "Cliente Descreve Eventos Diversos" (CDESDIV) foi então realizado. Por último, foram criadas categorias para a análise das verbalizações do terapeuta classificadas como "Explicações" e "Conselhos". As categorias construídas, assim como as figuras referentes ao percentual de ocorrência de cada categoria criada constam na próxima seção deste trabalho. 

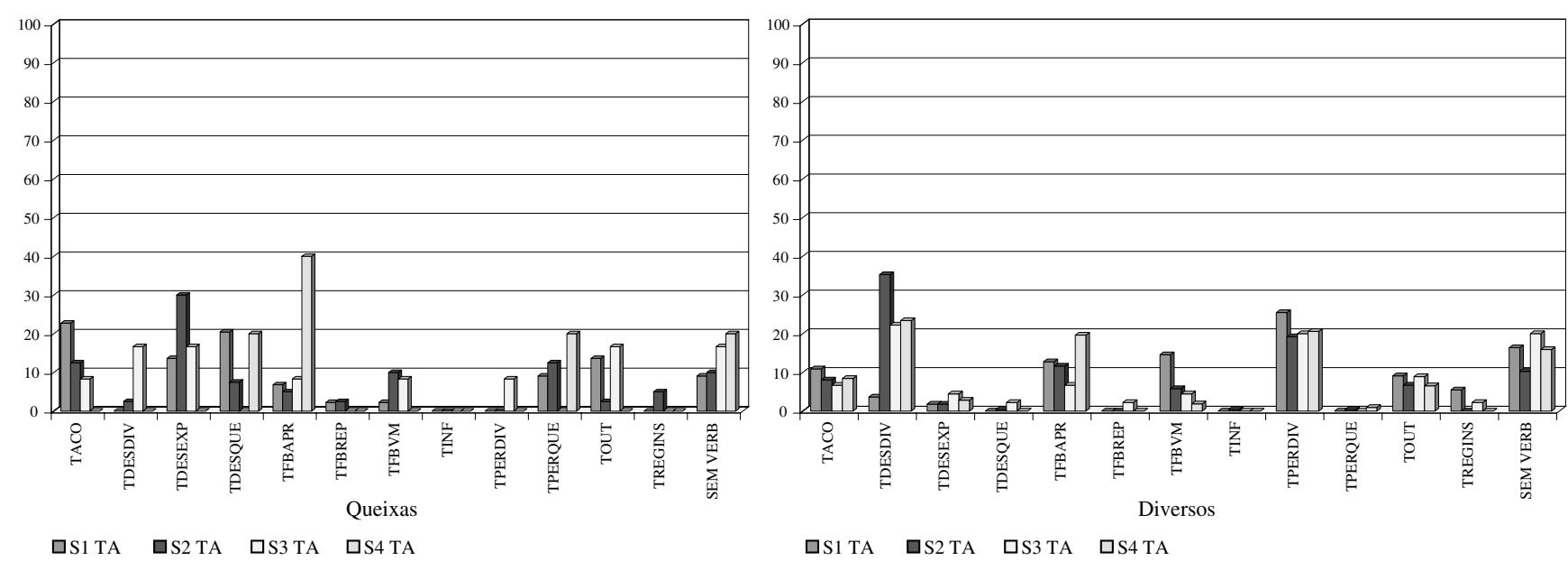

Figura 1. Percentual de ocorrência das categorias de registro de verbalizações do terapeuta A que se seguiram a verbalizações do cliente A sobre sua queixa e sobre eventos diversos.

\section{Resultados}

\section{$O$ andamento das sessões}

Na Figura 1, apresenta-se a ocorrência (em\%), por sessão, das categorias de registro de verbalizações do terapeuta A que se seguiram às verbalizações do cliente A sobre sua queixa e sobre eventos diversos.

Foram registradas 101 verbalizações do cliente A "Descrição sobre a Queixa" (CDESQUE), sendo 44, 40, 12 e cinco ocorrências em cada uma das sessões, respectivamente. $\mathrm{O}$ terapeuta A respondeu a estas verbalizações principalmente com "Explicação" (TDEXEXP - 0 a 30\%) e "Aconselhamento" (TACO - 0 a 23\%), ou não respondeu a essas verbalizações (8 a 19\%). A verbalização "Aconselhamento" decresceu de $22 \%$, na primeira sessão para zero na quarta sessão. O oposto aconteceu com as falas do cliente A sobre a queixa que não foram seguidas por verbalização do terapeuta, que ocorreram em $8 \%$ das oportunidades na sessão 1 e na sessão 4 chegaram a 19\%. As outras categorias de verbalizações do terapeuta ocorreram após verbalizações do cliente categorizadas como CDESQUE com percentual inferior a $20 \%$ em todas as sessões. Embora tenha havido um percentual relativamente alto de verbalizações de aprovação (TFBAPR) em seguida a falas do cliente relacionadas à queixa, este índice diz respeito a um número de ocorrências muito pequeno - principalmente na quarta sessão, na qual ocorreram somente cinco verbalizações de queixa.

Já com relação a descrição de eventos diversos do cliente A foram registradas 431 verbalizações "CDESDIV", sendo 55, 224, 45, e 107 ocorrências em cada uma das sessões, respectivamente. As verbalizações do terapeuta A que se seguiram a estas falas que ocorreram em maior percentual foram de "Descrição" e "Perguntas", também sobre eventos diversos (TDESDIV variou de $4 \%$ a $35 \%$ e TPERDIV variou de $19 \%$ a $25 \%$ de ocorrências). De 10 a $20 \%$ das ocorrências das descrições de eventos diversos por parte do cliente A não foram seguidas por qualquer verbalização do terapeuta (SEM VERB) e 6 a 19\% foram seguidas por verbalizações de "Aprovação"(TFBAPR).
Nas sessões do terapeuta A, as verbalizações do terapeuta com maior percentual de ocorrência após "Cliente Descreve Diversos" foram verbalizações categorizadas como "Perguntas" e "Descrições" sobre "Eventos Diversos". Já as verbalizações do cliente A sobre queixa foram preferencialmente seguidas por "Aconselhamento" e "Explicação" ou não foram seguidas por verbalização do terapeuta. Podemos notar, portanto, que o terapeuta A dispensou atenção diferenciada a verbalizações relacionadas à queixa e verbalizações sobre outros eventos.

A partir dos resultados relativos ao desempenho do terapeuta A e seu cliente foram plotadas as frequências acumuladas das verbalizações do terapeuta e do cliente relacionadas a queixas e eventos diversos, assim como as verbalizações mais freqüentes do terapeuta - "Terapeuta Aprova" (TFBAPR) e "Terapeuta Aconselha" (TACO) na segunda sessão (sessão 2 TA). O resultado está apresentado na Figura 2.

Pode-se observar na Figura 2 como estas verbalizações foram ocorrendo ao longo da sessão: o cliente A descreve vários assuntos (CDESDIV) durante quase toda a sessão, enquanto as descrições relacionadas à queixa (CDESQUE) ocorrem de maneira irregular e em muito menor frequência. As verbalizações do terapeuta A, por sua vez, são predominantemente de "Perguntas sobre eventos Diversos" (TPERDIV) e de "Aprovação" (TFBAPR), em geral subseqüentes a verbalizações do cliente sobre "Eventos Diversos" (CDESDIV). Considerando que o cliente estava em atendimento há mais de um ano, o padrão de responder do cliente $\mathrm{A}$ - ocorrência quase contínua de verbalizações sobre eventos não relacionados à queixa - poderia ser efeito de um procedimento de reforçamento diferencial por parte do terapeuta, hipótese fortalecida pelo padrão verbal diferenciado do terapeuta $\mathrm{A}$ em relação a falas de seu cliente.

Os percentuais de ocorrência de cada categoria de registro de verbalizações do terapeuta $B$ que se seguiram a verbalizações de descrição sobre eventos relacionados à queixa (CDESQUE) e a eventos diversos (CDESDIV) do cliente $\mathrm{B}$, em cada uma das sessões são apresentados na Figura 3. 
S2 TA

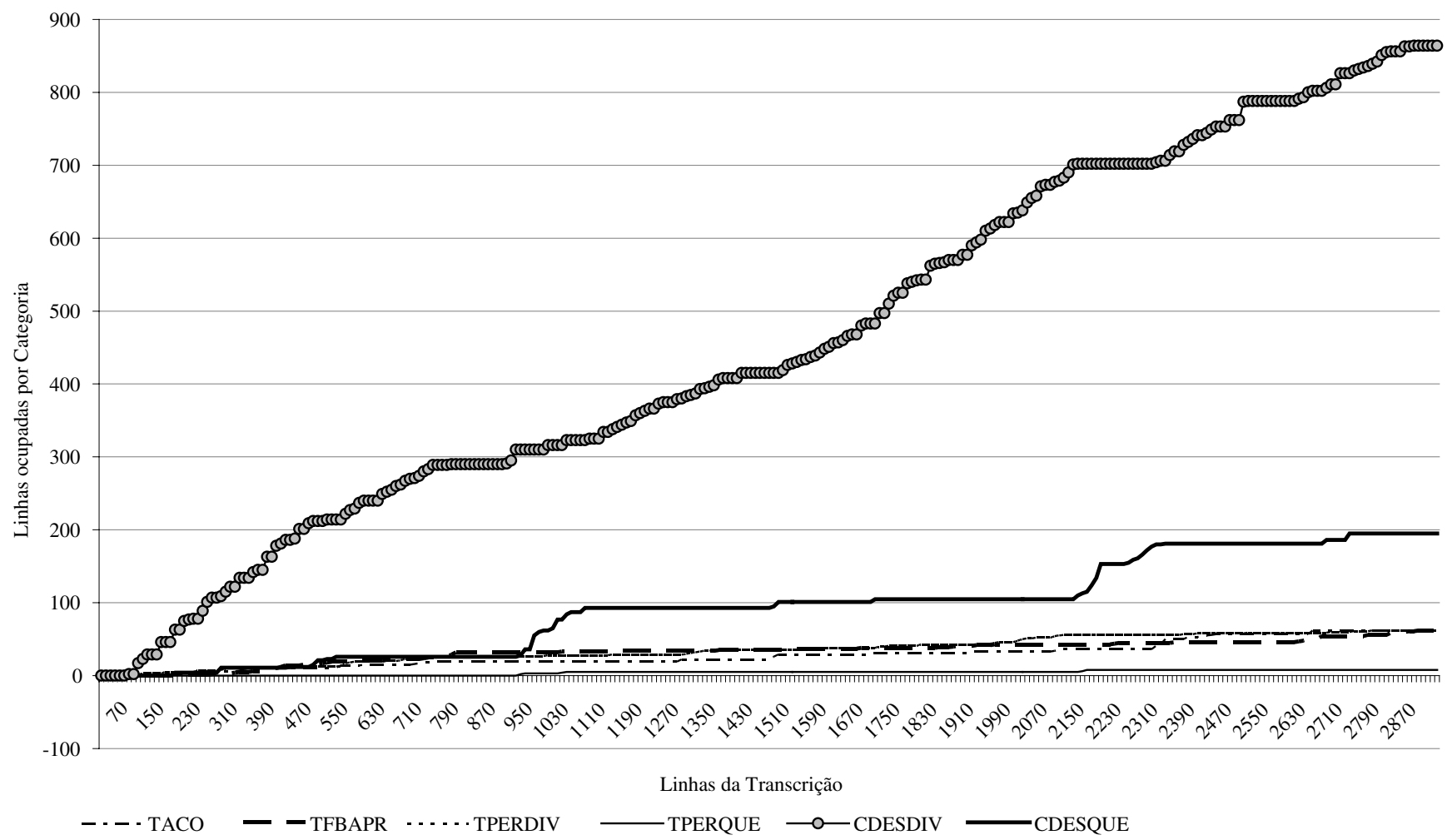

Figura 2. Frequiência acumulada de linhas da transcrição ocupadas por categoria do cliente e terapeuta na sessão 2 do terapeuta A.

O total de verbalizações de "Descrição sobre Eventos Relacionados à Queixa" nestas sessões foi 395 , das quais 384 foram seguidas por verbalizações do terapeuta e 11 por outras verbalizações do próprio cliente (menos que 5\%).

Na primeira sessão (S1 TB), as verbalizações do terapeuta que mais se seguiram a estas falas do cliente B foram categorizadas como "Verbalizações Mínimas" (TFBVM - 43\%), seguidas por "Perguntas sobre a queixa" (TPERQUE - 36\%). Já a partir da segunda sessão, verbalizações do cliente que descreviam a queixa foram seguidas preponderantemente por "Perguntas sobre Eventos Relacionados à Queixa" (TPERQUE - $70 \%$ a $80 \%$ ).

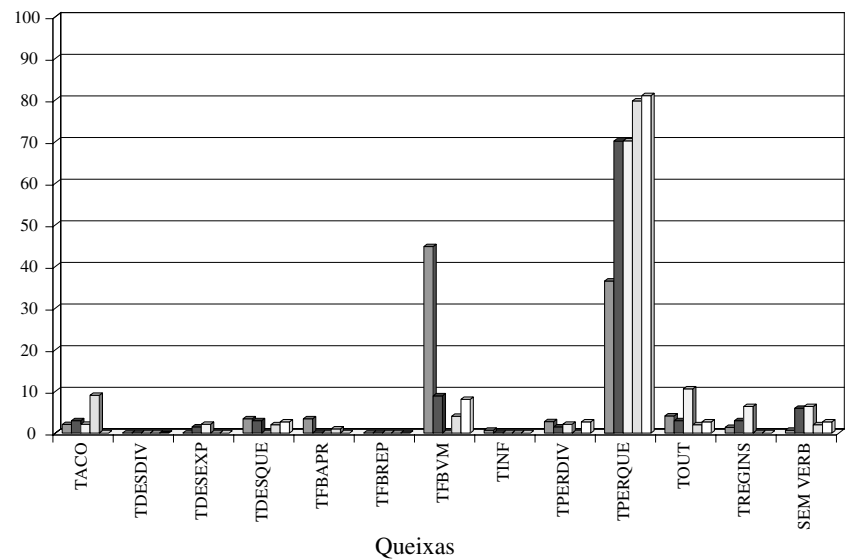

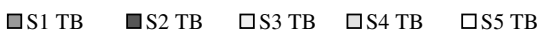

O total de verbalizações de descrição sobre eventos diversos (CDESDIV) do cliente B, nestas sessões, foi $120(25,20$, 10, 1 e 64 ocorrências, respectivamente), ou seja, bem menor ocorrência do que suas falas sobre a queixa. Na sessão 4 , a única fala do cliente classificada como "Descrição de Eventos Diversos" foi seguida por "Aconselhamento" (TACO). Já nas sessões 1 e 2, as verbalizações do terapeuta que mais freqüentemente se seguiram à "Descrição de Eventos Diversos" por parte do cliente (CDESDIV) foram "Perguntas sobre eventos diversos" (TPERDIV - $45 \%$ e $50 \%$ das verbalizações) e "Verbalizações Mínimas" (TFBVM - 31\% e 4\%). Na sessão 3 , o percentual de ocorrência de falas do terapeuta que se

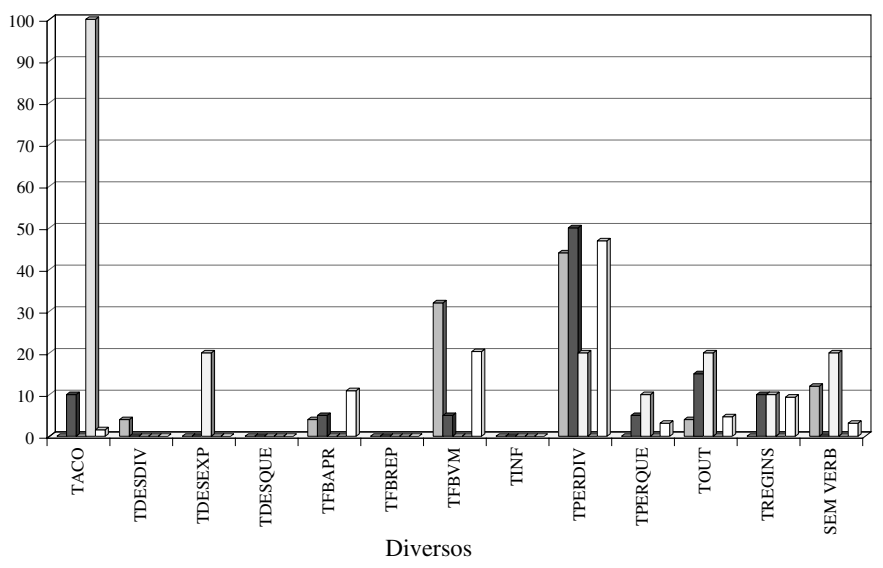

$\square \mathrm{S} 1 \mathrm{~TB} \quad \square \mathrm{S} 2 \mathrm{~TB} \quad \square \mathrm{S} 3 \mathrm{~TB} \quad \square \mathrm{S} 4 \mathrm{~TB} \quad$ 口S5 TB

Figura 3. Percentual de ocorrência das categorias de registro de verbalizações do terapeuta B que se seguiram a verbalizações do cliente B sobre sua queixa e sobre eventos diversos. 


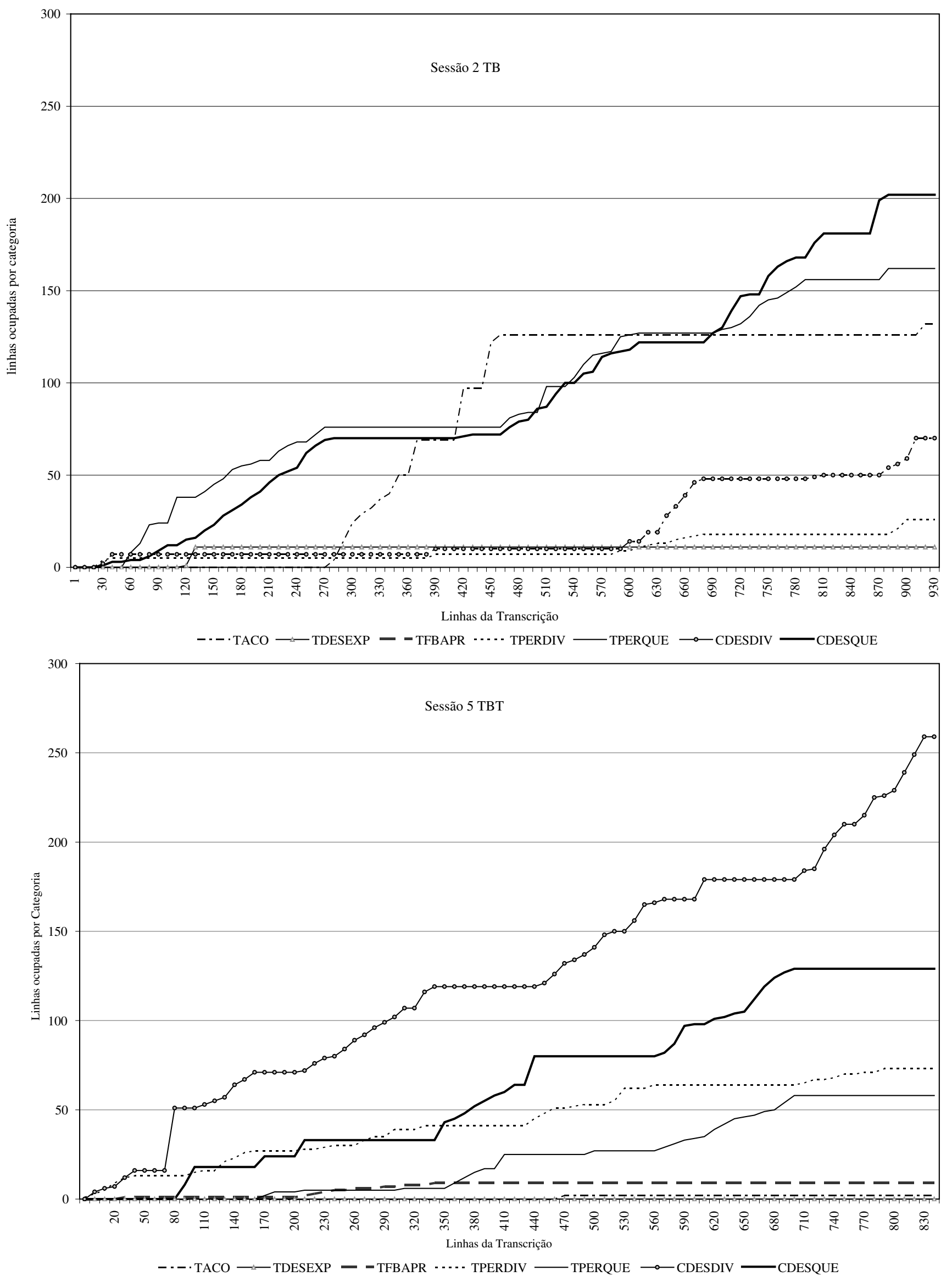

Figura 4. Frequiência acumulada de linhas da transcrição ocupadas por categoria do cliente e terapeuta nas sessões 2 e 5 do terapeuta B. 
Quadro 2. Categorias relativas a sequiências inicialmente categorizadas como "Terapeuta Aconselha o Cliente" e "Terapeuta Descreve Relações Explicativas ou Causais"

\begin{tabular}{|c|c|c|}
\hline & Categoria de Análise & Descrição \\
\hline \multirow{7}{*}{ 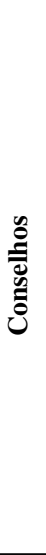 } & Solução de Problemas (SOLPROBLEMA) & Terapeuta sugeria alternativas de resposta a uma situação-problema. \\
\hline & $\begin{array}{l}\text { Terapeuta propõe atividade incompatível com a } \\
\text { resposta-queixa (ATIVIDADE) }\end{array}$ & $\begin{array}{l}\text { O terapeuta propunha que o cliente se engajasse em atividades (ou emitisse respostas) } \\
\text { - supostamente - incompatíveis com o problema-queixa. }\end{array}$ \\
\hline & $\begin{array}{l}\text { Exposição e/ou prevenção de respostas } \\
\text { (EXPOSIÇÃO): }\end{array}$ & $\begin{array}{l}\text { (1) O terapeuta utilizava o procedimento de exposição com prevenção de respostas ou um } \\
\text { de seus componentes. (2) O terapeuta recomendava ao cliente que realizasse registros de seu } \\
\text { comportamento, aparentemente como parte do preparo para este procedimento. }\end{array}$ \\
\hline & Ensino de Procedimentos (ENSINOPROC): & O terapeuta instruía o cliente em procedimentos decorrentes da análise do comportamento. \\
\hline & Coleta de Dados (COLETADADO): & $\begin{array}{l}\text { O terapeuta solicitava ao cliente que realizasse observações e/ou registros, de forma } \\
\text { sistemática ou assistemática, com exceção de registros para o desenvolvimento da exposição } \\
\text { com prevenção de respostas. }\end{array}$ \\
\hline & $\begin{array}{l}\text { Terapeuta recomenda que cliente se comporte } \\
\text { de modo a evitar ou expressar Eventos Privados } \\
\text { (PRIVADO): }\end{array}$ & O terapeuta solicitava ao cliente que alterasse pensamentos ou sentimentos ${ }^{5}$. \\
\hline & $\begin{array}{l}\text { Seqüência Insuficiente para classificação } \\
\text { (INSUFICIENTE): }\end{array}$ & $\begin{array}{l}\text { Seqüências cujo conteúdo era insuficiente para a identificação do tipo de aconselhamento } \\
\text { envolvido. }\end{array}$ \\
\hline \multirow{9}{*}{ 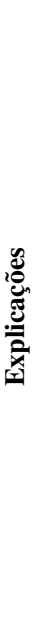 } & $\begin{array}{l}\text { Explicações baseadas em dados Demográficos } \\
\text { ou probabilísticos (DEMOGPROB): }\end{array}$ & $\begin{array}{l}\text { O terapeuta descrevia relações entre eventos com ênfase em dados demográficos ou } \\
\text { probabilísticos }{ }^{6} \text {. }\end{array}$ \\
\hline & $\begin{array}{l}\text { Aspectos fisiológico como causa } \\
\text { (FISIOLCAUSA): }\end{array}$ & $\begin{array}{l}\text { O terapeuta descrevia relações que recorrem a aspectos fisiológicos como agentes causadores } \\
\text { do comportamento. }\end{array}$ \\
\hline & Funcionamento do organismo (FUNCORGAN): & $\begin{array}{l}\text { O terapeuta descrevia o funcionamento do organismo, sem atribuição causal do } \\
\text { comportamento. }\end{array}$ \\
\hline & História como critério (HISTORIA): & $\begin{array}{l}\text { O terapeuta recorria à história passada do indivíduo para inferir a probabilidade de emissão } \\
\text { de determinada reposta por parte do cliente. }\end{array}$ \\
\hline & Diagnóstico como causa (DIAGNOST): & O terapeuta atribuía ao diagnóstico do cliente a causa de seu comportamento; \\
\hline & Eventos Internos como causa (INTERNA): & $\begin{array}{l}\text { O terapeuta atribuía a eventos internos, do tipo pensamentos ou sentimentos, a causa do } \\
\text { comportamento. }\end{array}$ \\
\hline & Descrição imprecisa (IMPRECISO): & $\begin{array}{l}\text { Seqüências na quais não era clara a relação descrita, devido a descrições imprecisas por parte } \\
\text { do terapeuta. }\end{array}$ \\
\hline & $\begin{array}{l}\text { Terapeuta aponta variáveis relevantes } \\
\text { (VARIÁVEIS): }\end{array}$ & $\begin{array}{l}\text { O terapeuta descrevia variáveis relevantes para o entendimento do comportamento, mas não } \\
\text { estabelecia a relação entre estas variáveis e o comportamento explicado. }\end{array}$ \\
\hline & $\begin{array}{l}\text { Explicações com ênfase em relações resposta- } \\
\text { consequiência (CONSEQUÊNCIA): }\end{array}$ & O terapeuta descrevia relações entre eventos com ênfase em relações resposta-conseqüência. \\
\hline
\end{tabular}

seguiram a falas do cliente do tipo CDESDIV foi distribuído uniformemente (20\% para cada categoria) entre as categorias "Explicação" (TDESEXP), "Perguntas sobre Eventos Diversos" (TPERDIV), "Outras Verbalizações" (TOUT) e por novas verbalizações do próprio cliente. "Perguntas sobre a Queixa" (TPERQUE) foram registradas em seguida a verbalizações do cliente sobre eventos diversos em $10 \%$ dos registros. Na sessão 5 , o padrão do terapeuta B foi muito semelhante ao seu padrão nas sessões 1 e 2 , no entanto, aumentaram as seqüências nas quais as falas do cliente B sobre temas diversos foram seguidas por "Aprovação" (TFBAPR), o que ocorreu em $10 \%$ das ocorrências.

A partir dos resultados relativos ao desempenho do terapeuta B e seu cliente foram "plotadas" as freqüências acumuladas das verbalizações do terapeuta e do cliente relacionadas a queixas e eventos diversos, assim como as verbalizações mais freqüentes do terapeuta - "Terapeuta

5 Esta categoria foi baseada em uma das categorias proposta por Martins (1999).

6 Não foram consideradas explicações baseadas em dados probabilísticos as verbalizações do terapeuta nas quais ele ressaltava a probabilidade de ocorrência de uma resposta a partir da identificação de uma determinada relação de contingência entre eventos. $O$ termo probabilístico aqui se refere a dados estatísticos.
Aprova" (TFBAPR) e "Terapeuta Aconselha" (TACO) na segunda e na quinta sessão. O resultado está apresentado na Figura 4.

Os padrões de verbalização de terapeuta e cliente $B$ foram bastante diferentes daqueles observados com o terapeuta e cliente A, conforme mostra a Figura 4. As classes de verbalização mais freqüentes já são um indicativo da diferença entre os terapeutas: enquanto nas sessões com o terapeuta A a categoria mais frequiente foi "Aprovação", para o terapeuta B houve mais verbalizações de "Aconselhamento" e "Explicação". Também foi bastante diferente o número de linhas ocupadas por falas do terapeuta: enquanto o terapeuta A teve falas muito curtas distribuídas ao longo de toda a sessão, o terapeuta B teve longos períodos de silêncio seguidos por falas muito prolongadas, chegando a superar o número de linhas de verbalizações do cliente.

Na quinta sessão do cliente B, no entanto, o padrão da sessão mudou sensivelmente. Nessa sessão, o terapeuta começou perguntando a respeito de eventos não relacionados à queixa (TPERDIV), e o cliente descreveu estes eventos (CDESDIV). O cliente, então, passou a queixar-se (a partir da linha 90), enquanto o terapeuta continuava a perguntar sobre outros eventos. A partir da linha 170 o terapeuta passou a perguntar sobre ambos - queixa e diversos (TPERDIV e TPERQUE) - e o cliente a descrever também ambos os eventos (CDESDIV 


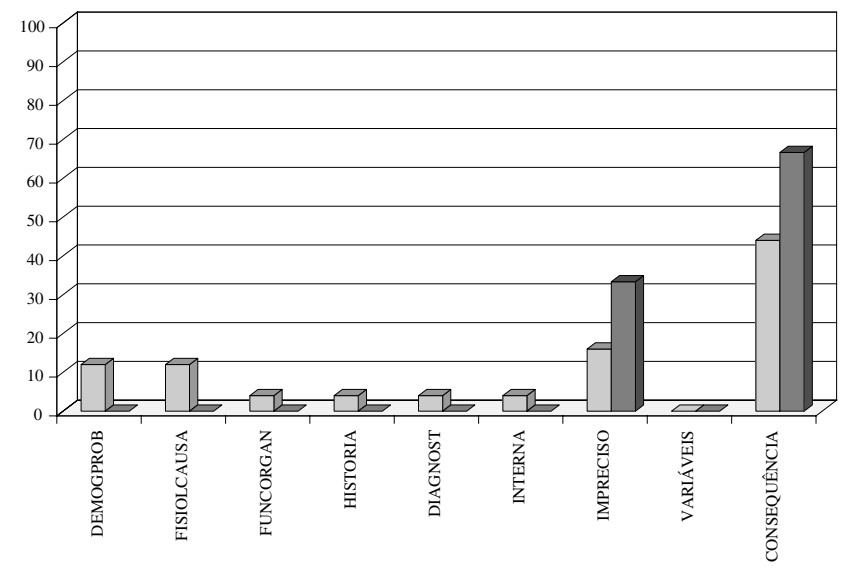

Sequências de verbalizações do tipo "EXPLICAÇÃO"

$\square$ TAT $\square$ TBT

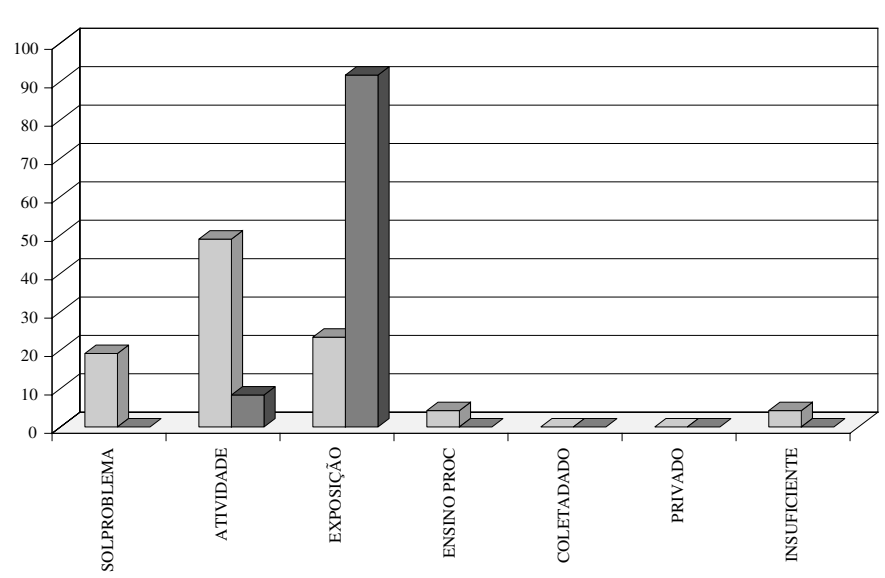

Sequências de Verbalizações do tipo "CONSELHOS"

$\square$ TAT $\square$ TBT

Figura 5. Percentual de ocorrências das categorias de análise dos segmentos categorizados como "Terapeuta Aconselha o Cliente" e "Terapeuta Descreve Relações Explicativas ou Causais".

e CDESQUE). Nota-se também a baixa ocorrência de explicações (TDESEXP) e conselhos (TACO), diferentemente do ocorrido na sessão 2. É possível observar, ainda, um aumento na ocorrência de verbalizações de aprovação (TFBAPR), com relação à sessão 2, principalmente no período inicial da sessão (até a linha 350), no qual foi maior a ocorrência de descrição de "Eventos Diversos" por parte do cliente.

Os resultados apresentados na Figura 4 sugerem que até a sessão 4, o terapeuta teria principalmente coletado dados a partir da queixa do cliente e, na sessão 5, teria alterado seu procedimento. A quinta sessão do terapeuta B e seu cliente, parece apresentar um padrão de interação intermediário entre o observado na sessão 2 desse mesmo terapeuta e aquele observado nas sessões do terapeuta $\mathrm{A}$ (Figura 2). Poder-se ia inferir, assim, que o terapeuta B, na sessão 5, estaria introduzindo um procedimento de reforçamento diferencial de verbalizações não relacionadas à queixa, semelhantemente ao que parece ocorrer com o terapeuta A. O aumento da ocorrência de verbalizações de aprovação na sessão 5 , em um período da sessão no qual era maior a ocorrência de descrições sobre eventos diversos poderia ser compreendido como parte deste procedimento de reforçamento diferencial. Entretanto, seria necessário acompanhar um maior número de sessões para confirmar essa tendência.

\section{A análise das explicações e aconselhamentos dos terapeutas}

A fim de analisar os tipos de "Explicação" (TDESEXP) e "Aconselhamento" (TACO) dos terapeutas, foram desenvolvidas o que aqui se convencionou chamar de categorias de análise de "seqüências de verbalizações". Para a elaboração dessas categorias, primeiramente foi realizado um levantamento de todas as ocorrências categorizadas como Explicações e Conselhos.

Em seguida, para cada ocorrência, foi realizada uma busca (na transcrição das sessões) da primeira verbalização do terapeuta ou do cliente na qual se observou uma mudan- ça de assunto para o tema que foi foco da explicação ou aconselhamento. Após identificar essa verbalização inicial, foi realizada a busca da última verbalização do terapeuta ou do cliente na qual se identificou o encerramento do tema. $\mathrm{O}$ trecho da transcrição compreendido entre a primeira e a última verbalização do tema que foi foco da explicação ou aconselhamento constituiu uma "Seqüência de Verbalizações". Assim, uma mesma seqüência de verbalizações podia conter mais de uma ocorrência da categoria de registro TDESEXP ou TACO.

A partir da identificação das sequiências de verbalizações, as seqüências relativas a TDESEXP e TACO foram, então, classificadas conforme consta no Quadro 2

Na Figura 5, apresenta-se o percentual de ocorrência de cada uma das categorias nas quais os terapeutas aconselharam (TACO) ou forneceram explicações (TDESEXP) a seus clientes. Nas sessões com o terapeuta $\mathrm{A}$ o número de ocorrências de conselhos por parte do terapeuta foi 132 segmentos (40, 35, 31 e 26, respectivamente). Os registros de categorias com percentual mais elevado foram "Terapeuta sugere atividade" (ATIVIDADE - 49\%), "Exposição com Prevenção de Respostas" (EXPOSIÇÃO - 21\%) e "Solução de Problemas" (SOLPROBLEMA - 19\%). Outras categorias ocorreram em menos de 5\% das sequiências. Já com relação às explicações por parte desse terapeuta, estas ocorreram em 48 segmentos $(12,24,6$ e 6 ocasiões em cada sessão, respectivamente). Pode-se observar na Figura 5 a prevalência de explicações do terapeuta A com ênfase nas conseqüências (Consequiência - 43\%), seguidas por explicações que recorriam a aspectos fisiológicos para explicar o comportamento (FISIOLCAUSA - $11 \%$ das ocorrências). Em 15\% das seqüências as explicações foram chamadas de IMPRECISAS, pois não se identificou o tipo de relação descrita. As demais categorias de explicação do terapeuta A ocorrem em menor frequiência, embora tenham ocorrido explicações de quase todas as categorias ao longo das sessões.

Nas sessões com o terapeuta B (S1 a S5 TB) ocorreram 34 segmentos categorizados como "Aconselhamento" $(3,12$, 2, 16 e 1 segmentos, respectivamente). Desses, $90 \%$ diziam 
respeito à preparação para o procedimento de exposição com prevenção de respostas (Exposição).

Já as explicações, por parte desse terapeuta, foram identificadas em quatro segmentos $(0,1,3,0$, e 0 ocasiões respectivamente), em 3 seqüências de verbalizações. Em duas delas o terapeuta descreveu relações do tipo consequiência entre eventos (CONSEQUÊNCIA) e em uma na qual não ficou clara a relação descrita (IMPRECISO).

Pode-se supor que o tipo de aconselhamento dado pelos terapeutas estava relacionado à queixa do cliente e ao procedimento terapêutico adotado pelo terapeuta. $\mathrm{O}$ terapeuta A aparentemente utilizou o procedimento de reforçamento diferencial de comportamentos incompatíveis com o comportamento-queixa. É coerente com esse procedimento a existência de poucos episódios de aconselhamento, enquanto se dava maior atenção à "conseqüenciação" (feedback) diferencial a diferentes respostas verbais do cliente. A ocorrência de um número superior de propostas de atividades incompatíveis com a resposta-queixa (ATIVIDADE) também parece relacionada a este tipo de procedimento. Além disso, o terapeuta A utilizou o procedimento de exposição com prevenção de respostas - aparentemente de modo assistemático. Já os conselhos do terapeuta B foram, em sua grande maioria, voltados para a coleta de informações, o que tipicamente fazem parte da preparação para o desenvolvimento do procedimento de exposição com prevenção de respostas. Entretanto, os dados da quinta sessão sugerem a adoção de um outro procedimento.

Ambos os terapeutas deram explicações com ênfase na relação resposta-consequiência, o que indicaria uma atuação consistente com os princípios teóricos da análise do comportamento. Por outro lado, a ocorrência de explicações imprecisas foi verificada com ambos os terapeutas, o que pode, no entanto, ser devido às condições de registro, que não permitiram o acesso do pesquisador a outros comportamentos verbais (não vocais). No que se refere a explicações, houve maior variabilidade no comportamento do terapeuta $A$, o que poderia apontar problemas com relação à sua consistência teórica - principalmente no que se refere a eventos privados e a diagnóstico como causa do comportamento. Entretanto, essas explicações podem envolver uma tentativa, por parte do terapeuta, de utilizar termos que o cliente compreenda, o que revela uma fragilidade do dado baseado apenas na observação do comportamento verbal.

\section{Discussão}

Os resultados encontrados nesse trabalho podem ser interpretados, na tentativa de caracterizar o trabalho dos terapeutas estudados, segundo os critérios propostos por Hayes (1991) e Meyer (1995).

A respeito do critério tecnológico, Meyer (1995) e Banaco (1999) salientam que o uso indiscriminado de uma técnica, desvinculada de outros níveis de análise seria incoerente com as propostas da análise do comportamento. Da mesma forma, as técnicas empregadas por um terapeuta comportamental deveriam ser derivadas de princípios do comportamento. Diversos aspectos referentes às sessões terapêuticas analisadas sugerem que a aplicação de técnicas, para estes terapeutas, não foi desvinculada de outros níveis de análise.
Ambos os terapeutas optaram por procedimentos nãoaversivos, como sugeriu o baixo percentual de verbalizações de reprovação por parte dos terapeutas nas sessões analisadas. A escolha pela não utilização de punição é uma característica importante na prática de um analista do comportamento, como indicado pela adoção de procedimentos baseados em reforçamento positivo especialmente defendida por Skinner (1953/1993, 1968/1975, 1974/1992, 1989/1995) e Sidman (1995).

Já os desempenhos dos terapeutas eram diferentes entre si, apesar de os clientes terem chegado à terapia com diagnósticos semelhantes, de acordo com o DSM-IV. No entanto, a atuação desses terapeutas pareceu atender às necessidades específicas para o manejo das queixas apresentadas pelos seus respectivos clientes. Por exemplo, o terapeuta A parece ter utilizado basicamente um procedimento de "conseqüenciação diferencial" de verbalizações do cliente não relacionadas à queixa (Reforçamento Diferencial de Outros Comportamentos - DRO), embora tenham ocorrido um percentual alto de falas de "aconselhamento". O cliente A, no caso, apresentava uma ação "lentificada" no ambiente e dificuldade em iniciar respostas "espontaneamente", o que provavelmente exigia que o terapeuta evocasse novas respostas por meio de conselhos e sugestões, ou mesmo, realizando as atividades juntamente com o cliente e, paralelamente, reforçasse qualquer relato ou ação direta contrária à queixa. $\mathrm{O}$ terapeuta $\mathrm{B}$, por sua vez, enfatizou em suas primeiras sessões a investigação da queixa do cliente. As perguntas por ele formuladas foram, em boa parte, voltadas para aspectos relativos à resposta obsessivo-compulsiva - antecedentes médicos, agentes desencadeantes, pensamentos e sentimentos que acompanham etc. Seu desempenho envolveu, na maior parte do tempo, verbalizações de aconselhamento e explicação, o que indicaria que ele utilizou preferencialmente a descrição de relações funcionais por meio de regras e conselhos como estratégia terapêutica. Ambos os tipos de desempenho podem ser encontrados em trabalhos relatados na literatura sobre terapia comportamental (Guilhardi \& Queiroz, 1997; Kohlenberg \& Tsai, 1991).

As diferenças nos padrões de atendimento dos dois terapeutas estudados apontam para uma questão bastante controversa na análise do comportamento, relativa a qual é a melhor forma de produzir mudanças comportamentais em psicoterapia. Alguns autores (por ex. Catania, 1992) defendem que o controle por regras é uma forma eficaz de controle do comportamento humano. Catania (1992), por exemplo, defende que a modelagem do comportamento verbal aumenta a probabilidade de mudança no comportamento não verbal correspondente. Guedes (1993), embora reconheça a importância das regras na produção de novas respostas, defende que a obtenção de respostas novas por meio de conselhos ou regras é mais provável com pacientes cujo repertório de comportamentos de "seguir regras" é bastante fortalecido e que clientes que não apresentem esse tipo de repertório trariam mais dificuldade para o uso desse recurso. Por sua vez, a ênfase no feedback diferencial, que modelaria e manteria certos padrões de comportamento durante a sessão, pode esbarrar em questões de generalização e até mesmo na discussão de como e em que circunstâncias o comportamento verbal fortalecido na sessão controlaria 
comportamento não verbal fora dela. Novos estudos poderiam verificar experimentalmente as vantagens e limitações de cada um dos procedimentos.

Os resultados sugerem que ambos os terapeutas utilizaram análise de contingências - ou análise funcional. Essa hipótese pode ser, em parte, inferida dos tipos de explicação que ocorreram com maior frequiência em todos os atendimentos - "explicações com ênfase em relações resposta-consequência". A existência de explicações classificadas como explicações com ênfase na relação resposta-consequiência também permite inferir que a atuação dos terapeutas estaria sob controle do repertório do cliente em cada situação apresentada e não apenas sob controle de determinado diagnóstico psiquiátrico. Esses resultados parecem indicar que os terapeutas trabalharam de maneira que poderia ser avaliada como atendendo ao que Meyer (1995) chamou de critério metodológico do trabalho.

A análise dos tipos de explicações e conselhos de ambos os terapeutas teve como objetivo investigar principalmente a adequação dos procedimentos adotados e das relações estabelecidas pelos terapeutas entre eventos ambientais e o responder do cliente. Ambos os terapeutas parecem ter utilizado, em diferentes momentos, diferentes recursos explicativos na análise dos problemas que lhes eram apresentados. A grande maioria das verbalizações analisadas referia-se a relações causais ou explicativas consistentes com os pressupostos conceituais e filosóficos da análise do comportamento. Houve, no entanto, verbalizações nas quais ambos os terapeutas recorreram a explicações que poderiam indicar uma perspectiva filosófica incompatível com a análise do comportamento.

Pode-se considerar, entretanto, que explicações que enfatizassem outros agentes causais na determinação do comportamento - desde que este não fosse o recurso explicativo exclusivo, ou quase exclusivo, por parte do terapeuta - não implicariam necessariamente em inconsistência teórica. Skinner (1989/ 1995) identificou três grandes determinantes do comportamento - o filogenético, ontogenético e o cultural. Explicações baseadas em eventos fisiológicos, demográficos ou probabilísticos, históricos, ou até mesmo eventos internos, podem, ou não, estar relacionadas a um ou mais destes três níveis de determinação do comportamento, a depender do tipo de relação estabelecida e da exclusividade ou não da explicação com relação a outros níveis de análise.

Os desempenhos dos terapeutas estudados são uma amostra das possibilidades de aplicação do conhecimento desenvolvido por terapeutas comportamentais no contexto clínico. Pode-se observar que, embora ambos tenham tido uma atuação compatível com a proposta analítica-comportamental, há diferenças importantes entre os terapeutas. Essas diferenças evidenciam a imprecisão da análise de contingências enquanto instrumento conceitual para a análise do caso clínico. A variabilidade de análises que podem ser derivadas de um problema específico, bem como as diferentes possibilidades de derivação de um tratamento a partir dessa análise, dificultam consideravelmente a formação do terapeuta analista do comportamento. A pesquisa mostra-se então necessária como um meio para o aperfeiçoamento dos instrumentos e métodos disponíveis, em busca de uma prática menos baseada no conhecimento tácito e mais empiricamente fundamentada.

\section{Referências}

American Psychiatric Association (1995). Manual diagnóstico e estatístico de transtornos mentais. (D. Batista, Trad.) Porto Alegre: Artes Médicas. (Trabalho original publicado em 1994)

Banaco, R. A. (1999). Técnicas cognitivo-comportamentais e análise funcional. Em R. R. Kerbauy \& R. C. Wielenska (Org.), Sobre comportamento e cognição, Vol. 4, (pp. 75-82). Santo André: Arbytes.

Banaco, R. A. \& Zamignani, D. R. (2004). An analytical-behavioral panorama on the anxiety disorders. Em T. C. C. Grassi (Org.), Contemporary challenges in the behavioral approach: A Brazilian overview (pp. 9-26). Santo André: Esetec.

Barlow, D. H. (1999). Manual clínico dos transtornos psicológicos. (M. R. B. Osório, Trad.) Porto Alegre: Artmed.

Caballo, V. E. (2003). Manual para o tratamento cognitivocomportamental dos transtornos psicológicos. (M. de L. Pedro, Trad.) São Paulo: Santos.

Catania, A. C. (1992). Learning. Englewood Cliffs, NJ: Prentice Hall.

Freeston, M. H. \& Ladouceur, R. (2003). Análise e tratamento das obsessões. Em V. E. Caballo (Org.), Tratamento cognitivocomportamental dos transtornos psicológicos, (pp. 137-170). São Paulo: Santos Editora.

Guedes, M. L. (1993). Equívocos da terapia comportamental. Temas em Psicologia, 2, 81-85.

Guilhardi, H. J. \& Queiroz, P. B. P. S. (1997). A análise funcional no contexto terapêutico: O comportamento do terapeuta como foco da análise. Em M. Delitti (Org.), Sobre comportamento e cognição, Vol. 2, (pp. 45-97). Santo André: Arbytes.

Hayes, S. C. (1991) The limits of technological talk. Journal of Applied Behavior Analysis, 24, 417-420.

Kohlenberg, R. J. \& Tsai, M. (1991). Functional analytic psychotherapy: Creating intense and curative therapeutic relationships. New York: Plenum.

Martins, P. (1999). Atuação de terapeutas estagiários com relação a falas sobre eventos privados em sessões de psicoterapia comportamental. Dissertação de Mestrado, Universidade Federal do Pará, Belém.

Meyer, S. B. (1995) Quais os requisitos para que uma terapia seja considerada comportamental? Retirado em 04/2005, http:// www.inpaonline.com.br/artigos.

OMS, Organização Mundial de Saúde (1993). Classificação de transtornos mentais e de comportamento da CID-10: Descrições clínicas e diretrizes diagnósticas. (D. Caetano, Trad.) Porto Alegre: Artes Médicas.

Riggs, D. S. \& Foa, E. B. (1999). Transtorno obsessivo-compulsivo. Em H. David Barlow (Org.), Manual clínico dos transtornos psicológicos (pp. 217-271). Porto Alegre: Artmed.

Salkovskis, P. M. \& Kirk, J. (1997). Distúrbios obsessivos. Em K. Hawton, P. M. Salkovskis, J. Kirk \& D. M. Clark (Orgs.), Terapia cognitivo-comportamental para problemas psiquiátricos: Um guia prático (pp. 186-239). São Paulo: Martins Fontes.

Sidman, M. (1995). Coerção e suas implicações. (M. A. Andery \& T. M. Sério, Trads.) Campinas: Editorial Psy.

Skinner, B. F. (1975). Tecnologia do ensino. (R. Azzi, Trad.) São Paulo: Herder. (Trabalho original publicado em 1968)

Skinner, B. F. (1992). Sobre o behaviorismo. (M. da P. Villalobos, Trad.) São Paulo: Cultrix. (Trabalho original publicado em 1974) 
Skinner, B. F. (1993). Ciência e comportamento humano. (J. C. Todorov \& R. Azzi, Trads.) São Paulo: Martins Fontes. (Originalmente publicado em 1953).

Skinner, B. F. (1995). Questões recentes na análise comportamental. (A. L. Néri, Trad.) Campinas: Papirus. (Trabalho original publicado em 1989)

Vermes, J. S. \& Zamignani, D. R. (2002). A perspectiva analíticocomportamental no manejo do comportamento obsessivo- compulsivo: Estratégias em desenvolvimento. Revista Brasileira de Terapia Comportamental e Cognitiva, 4(2), 135-149.

Recebido em 14.05.2004

Primeira decisão editorial em 04.11.2004

Versão final em 13.12.2004

Aceito em 09.04.2005 